\title{
ESTUDO ESPECTROSCÓPICO DE VIDROS A BASE DE ALUMINATO DE CÁLCIO CONTENDO Nd ${ }^{3+}$
}

\author{
Édison Pecoraro, Luiz Antonio de Oliveira Nunes* \\ Instituto de Física de São Carlos - Universidade de São Paulo - Av. Dr. Carlos Botelho, 1465 - 13560-250 - São Carlos - SP \\ Jurací A. Sampaio, Sérgio Gama \\ Instituto de Física Gleb Wataghin - Universidade Estadual de Campinas - Unicamp - 13083-970 - Campinas - SP \\ Mauro L. Baesso \\ Departamento de Física - Universidade Estadual de Maringá - Av. Colombo, 5790 - 87020-900 - Maringá - PR
}

Recebido em 8/9/98; aceito em 22/11/99

\begin{abstract}
SPECTROSCOPIC STUDY OF CALCIUM ALUMINATE-BASED GLASSES WITH Nd ${ }^{3+}$. Multicomponent ( $\mathrm{Al}_{2} \mathrm{O}_{3}, \mathrm{CaO}, \mathrm{SiO}_{2}, \mathrm{MgO}$ ) calcium aluminate-based glasses containing $\mathrm{Nd}^{3+}$ were $^{3+}$ prepared in order to evaluate their possibilities as laser host materials. The refractive index, UV-visible-near IR absorption spectrum, IR and visible luminescence spectra, and fluorescence decay time were measured. Judd-Ofelt model was used to obtain experimental intensity parameters $\left(\Omega_{2}, \Omega_{4}\right.$ and $\Omega_{6}$ ), emission cross-section, radiative lifetimes, emission branching ratios and quantum efficiency.
\end{abstract}

Keywords: lasers; calcium-aluminate glasses; neodymium.

\section{INTRODUÇÃO}

Os vidros a base de aluminato de cálcio são estudados desde a década de $40^{1}$ devido às suas excelentes características mecânicas e ópticas. $\mathrm{O}$ sistema binário $\mathrm{CaO}-\mathrm{Al}_{2} \mathrm{O}_{3}$ apresenta transmissão na região do infravermelho até $6 \mu \mathrm{m}^{1}$. Todavia este sistema possui a tendência de devitrificar-se facilmente. Tal problema é geralmente contornado adicionando-se à composição do vidro, óxidos que aumentam sua estabilidade frente à cristalização (geralmente óxidos de metais alcalinos ou alcalino terrosos). A adição destes óxidos pode causar redução da transmissão na região do infravermelho. Essa redução é devido ao fato de que, geralmente são usados óxidos de metais leves. $\mathrm{Na}$ tentativa de obter-se um equilíbrio entre a transmissão e a estabilidade, são também adicionadas pequenas quantidades de $\mathrm{SiO}_{2}$ e outros óxidos, obtendo-se assim, vidros a partir de formadores de rede não convencionais.

Como na maioria dos vidros óxidos, pode-se observar no sistema aluminato de cálcio o aparecimento de uma banda de absorção muito pronunciada causada pela presença do grupo

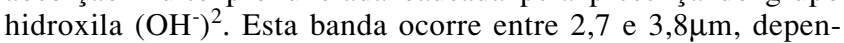
dendo da composição da matriz e reduz significativamente a transmissão nesta região espectral. Ela pode ser evitada preparando-se a amostra sob vácuo. Com este procedimento Davy ${ }^{2}$ obteve transmissão no infravermelho próxima à da safira $\left(\mathrm{Al}_{2} \mathrm{O}_{3}\right)(5 \mu \mathrm{m})$ que, quando dopada com $\mathrm{Cr}^{3+}$, é usada como meio ativo de laseres (laser de rubi).

Os vidros a base de aluminato podem ser utilizados no desenvolvimento de componentes ópticos que necessitam operar em ambientes hostis, como atmosferas corrosivas, altas temperaturas, etc. Eles são concorrentes diretos dos vidros fluoretos que, apesar de possuírem energia de fônons mais baixas $\left(500 \mathrm{~cm}^{-1}\right)^{3}$, apresentam baixa resistência à tração e ao choque térmico, além de sofrerem corrosão pela água ${ }^{4}$. Como exemplo, os vidros aluminatos estudados nesse trabalho apresentam dureza de Vickers de $\cong 800 \mathrm{MPa}(\cong 250 \mathrm{MPa}$ para vidros fluoretos) e temperatura de transição vítrea (TG) de $750^{\circ} \mathrm{C}^{5}$ $\left(\cong 250^{\circ} \mathrm{C}\right.$ para vidros fluoretos).

Uma outra aplicação possível para os vidros aluminato é a

*e-mail: luizant@ifqsc.sc.usp.br de meio ativo na obtenção de laseres na região do infravermelho. Para isso, geralmente são inseridos nas matrizes íons terras raras $3+(\mathrm{TR})$, como no caso deste trabalho o $\mathrm{Nd}^{3+}$. Este íon possui absorção na região de $800 \mathrm{~nm}\left({ }^{4} \mathrm{I}_{9 / 2} \rightarrow{ }^{4} \mathrm{~F}_{5 / 2}\right)$, o que possibilita a construção de um laser bombeado por laser de diodo. Um exemplo disto é a utilização em telecomunicações de laseres de Nd-YAG (yttrium alluminum garnet ou granada de ítrio e alumínio) ou Nd-YLF (yttrium lithium fluoride ou fluoreto de lítio e ítrio), bombeados por laser de diodo (InGaAsP com emissão em $800-820 \mathrm{~nm})^{6} \mathrm{O} \mathrm{Nd}^{3+}$ possui uma emissão em $\cong 1300 \mathrm{~nm}$, que pode ser empregada como fonte de sinal para fibras de sílica, as quais possuem uma janela de transmissão neste comprimento de onda. Laseres pulsados de alta potência também podem ser construídos empregando os vidros a base de aluminato de cálcio, pois devido às suas propriedades mecânicas ${ }^{7}$, ele suportaria a alta densidade de energia de bombeio necessária nestes tipos de laseres.

Neste artigo são mostrados os resultados da investigação de sistemas vítreos quaternários a base de aluminato de cálcio com baixa quantidade de $\mathrm{SiO}_{2}$, contendo íons $\mathrm{Nd}^{3+}$ em diversas concentrações. O objetivo é avaliar a possível utilização destes vidros como meio ativo para laseres no IV.

\section{PARTE EXPERIMENTAL}

A composição de partida (\% em massa), foi baseada na proposta por Davy et $\mathrm{al}^{2}:(47,4 \%) \mathrm{CaO}+(41,5 \%-\mathrm{x} \%) \mathrm{Al}_{2} \mathrm{O}_{3}+$ $(7,0 \%) \mathrm{SiO}_{2}+(4.1 \%) \mathrm{MgO}+\mathrm{x} \% \mathrm{Nd}_{2} \mathrm{O}_{3}$, onde $\mathrm{x}=0,5 ; 1,0 ; 1,5$; 2,$0 ; 2,5 ; 3,0 ; 3,5 ; 4,0 ; 4,5$ e $5,0 . \mathrm{O} \mathrm{Nd}_{2} \mathrm{O}_{3}$ substitui o $\mathrm{Al}_{2} \mathrm{O}_{3}$. Foi observado durante o preparo das amostras que para concentrações acima de 5,0\% ocorre cristalização. Atribuiu-se o acrônimo CASM para designar os vidros obtidos neste traba1ho. As amostras foram preparadas em um forno marca Varian/ NRC, modelo 2835. A fusão das mesmas foi feita sob vácuo em cadinho de grafite a temperatura de $1500^{\circ} \mathrm{C}$ por aproximadamente 5 horas. O quenching (resfriamento por choque térmico), foi realizado movendo-se o cadinho para uma região mais fria do forno.

Os espectros de absorção foram obtidos utilizando um Espectrofotômetro Cary 17 e um Espectrômetro Nicolet FT-IR mod. Magna 850. Os espectros de luminescência no infravermelho foram obtidos utilizando um laser $\mathrm{Ar}^{+}$Coherent Innova 400 (linha 
em 514nm) como fonte de bombeio, o sinal de luminescência foi coletado por um sistema composto por um Monocromador (CzeniTuner) Thermo Jarrel Ash 0,30m, um detector de Ge (não refrigerado), um modulador Stanford SRS mod. SR540, um Lock-in EG\&G PAR mod. 124A. A digitalização do sinal foi realizada por um sistema de aquisição de dados construído em nosso laboratório. Nas medidas de tempo de vida do nível ${ }^{4} \mathrm{~F}_{3 / 2}$, foi empregada a mesma montagem e o sinal foi diretamente digitalizado por um Osciloscópio Digital HP 54501A 100MHz, que estava interfaciado a um microcomputador. Nas medidas a $77 \mathrm{~K}$, foi utilizado um criostato de dedo frio. Os índices de refração das amostras foram medidos com um Refratômetro de Abbe Carl Zeiss Jena mod. Universal.

\section{RESULTADOS E DISCUSSÃO}

\section{Absorção}

A matriz do vidro CASM apresenta transmissão no IV até $4,5 \mu \mathrm{m}$ (Figura 1) e índice de refração de 1,65. A transmissão no infravermelho está relacionada com a energia máxima de fônons da rede, (que depende da composição do material), e com a natureza das ligações químicas entre seus constituintes. Para que a transmissão no infravermelho apresente deslocamento para comprimentos de onda maiores, é necessário a força de atração entre os íons ser fraca e a massa dos mesmos alta $^{1}$. No caso da dependência com a composição, o elemento que influencia mais no valor final da transmissão é aquele que está presente em maior quantidade e com maior peso atômico. No caso dos vidros CASM, estes são o alumínio e o oxigênio. A transmissão da matriz vítrea pode se estender mais na região do IV, como no caso dos vidros fluoretos a base de metais pesados (HMF), onde elementos como o índio, chumbo, cádmio, etc, diminuem a energia de fônons da rede para valores de aproximadamente $500 \mathrm{~cm}^{-13}$.

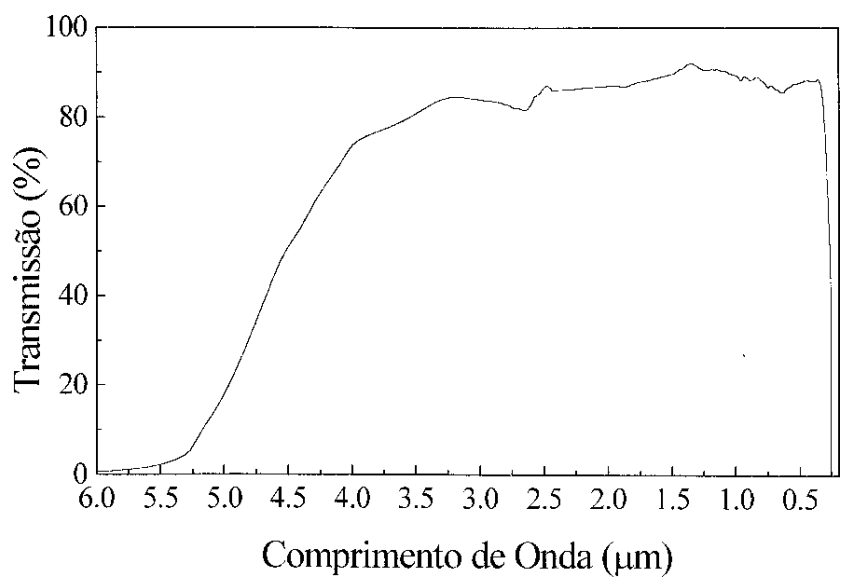

Figura 1. Espectro de transmissão na região do IV da matriz do vidro CASM (amostra com 0,4cm de espessura, 300K).

A Figura 2 mostra o espectro de absorção UV-Vis.-IV de uma amostra do vidro CASM com $5 \%$ de $\mathrm{Nd}_{2} \mathrm{O}_{3}$ a $300 \mathrm{~K}$. Observam-se as transições características do íon $\mathrm{Nd}^{3+8}$. Os espectros de todas as amostras apresentam bandas largas devido à presença dos íons TR em uma matriz vítrea. Neste caso há ordem apenas de curto alcance e o alargamento das linhas é do tipo inomogêneo.

\section{Judd-Ofelt ${ }^{9,10}$}

A teoria de Judd-Ofelt é largamente empregada na análise quantitativa das transições f-f dos íons TR em vidros ${ }^{11,12} \mathrm{e}$ cristais ${ }^{13,14}$. Judd e Ofelt consideram que as transições f-f

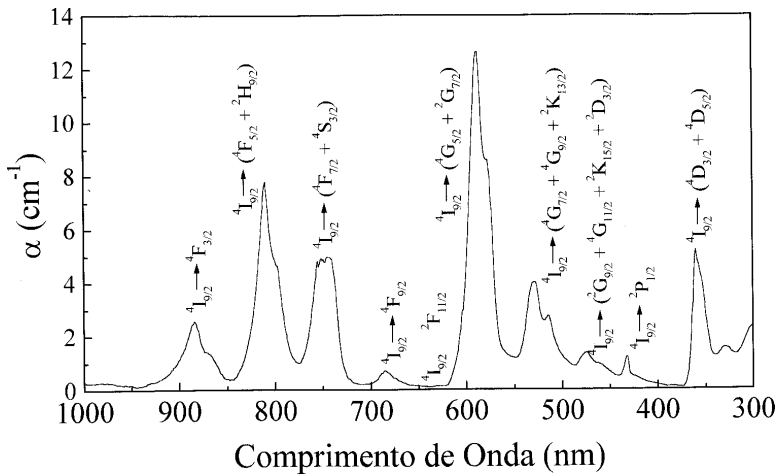

Figura 2. Espectro de absorção do vidro CASM contendo $5 \%$ de $\mathrm{Nd}_{2} \mathrm{O}_{3}$ (amostra com 0,3cm de espessura, 300K).

podem ser descritas numa soma de poucos termos, que leva em consideração a contribuição dos mecanismos de dipolo elétrico forçado e dipolo magnético:

$$
\begin{aligned}
& F_{\text {calc }}=\frac{8 \pi^{2} m}{3 h e^{2} n^{2}} \frac{v}{(2 J+1)}\left[e^{2} \frac{n\left(n^{2}+2\right)^{2}}{9} \sum_{\lambda=2,4,6} \Omega_{\lambda}\left|\left\langle a J\left\|U^{\lambda}\right\| b J^{\prime}\right\rangle\right|^{2}\right. \\
& \left.+n^{3}\left(\frac{e h}{2 m c}\right)^{2}\left|\left\langle a J\|L+2 S\| b J^{\prime}\right\rangle\right|^{2}\right]
\end{aligned}
$$

sendo, $F_{\text {calc }}$ a força de oscilador calculada; $v$ a freqüência da transição $\left(\mathrm{s}^{-1}\right) ; h$ a constante de Planck; $J$ o número quântico de momento angular total do estado fundamental; $J^{\prime}$ o número quântico de momento angular total do estado excitado; $\boldsymbol{U}^{\lambda} \mathrm{o}$ operador tensorial unitário; $L+2 S$ o operador de dipolo magnético; $m$ a massa do elétron; $e$ a carga do elétron; $\left(n^{2}+2\right)^{2} / 9 n$ o fator de correção de Lorentz, e $n$ é o índice de refração do meio. $\mathrm{O}$ primeiro termo dentro dos colchetes representa a intensidade de linha para mecanismos de dipolo elétrico e o segundo termo, descreve a intensidade de linha para o mecanismo de dipolo magnético. $\Omega_{\lambda}$ são os parâmetros de intensidade de Judd-Ofelt, com $\lambda$ valendo 2,4 e 6 . Esses parâmetros podem ser determinados experimentalmente, através das forças de oscilador obtidas a partir do espectro de absorção (Figura 2). A expressão para a força de oscilador experimental é dada por:

$$
F \exp =\frac{m c}{\pi e^{2} N} \int \alpha(v) d v
$$

sendo, $c$ a velocidade da luz e $N$ o número de íons por $\mathrm{cm}^{3}$ e o termo na integral é a área sob a banda da transição. Igualando-se as expressões (1) e (2), tem-se um sistema de equações super determinado que quando solucionado pelo método dos mínimos quadrados, dá como resultado os valores dos $\Omega_{\lambda}$.

As transições entre estados $f$ puros via mecanismo de dipolo elétrico são proibidas por paridade, enquanto que as por dipolo magnético são permitidas. As transições por dipolo elétrico ocorrem devido a mistura das funções de onda dos estados da configuração $4 \mathrm{f}^{\mathrm{n}}$ com estados de paridades opostas da configuração $4 \mathrm{f}^{\mathrm{n}-1} 5 \mathrm{~d}$. Esta mistura é causada pelos termos ímpares do campo cristalino. No caso do $\mathrm{Nd}^{3+}$, as transições por dipolo magnético são fracas, sendo que somente a ${ }^{4} \mathrm{I}_{9 / 2} \rightarrow{ }^{4} \mathrm{~F}_{9 / 2}$ foi observada e considerada. Nesse caso, a sua contribuição foi calculada segundo a equação proposta por Carnall ${ }^{15}$ :

$$
P_{m d}=P^{\prime} n
$$

sendo, $n$ o índice de refração do meio e $P^{\prime}$ a força de oscilador 
por dipolo magnético, calculada para íons lantanídios em solução aquosa.

O significado físico dos parâmetros de intensidade de JuddOfelt ainda hoje é assunto de controvérsias. Alguns autores consideram que tais parâmetros relatam a interação entre o campo ligante e os estados eletrônicos do íon TR, enquanto outros afirmam que eles não tem significado físico ${ }^{16}$. Apesar disso, a maioria dos autores atribui as variações nos valores dos parâmetros à mudanças no ambiente químico ao redor do íon TR. A Tabela 1 apresenta os valores de $\Omega_{2}, \Omega_{4}$ e $\Omega_{6}$ obtidos para o íon $\mathrm{Nd}^{3+}$ no vidro CASM. Não há variação dos valores $\operatorname{dos} \Omega_{\lambda}$ com a concentração de neodímio.

Tabela $1-\Omega_{\lambda}$ (parâmetros de intensidade); $A$ (probabilidade de transição) e $\beta$ (razão de ramificação) para o $\mathrm{Nd}^{3+}$ na matriz CASM. A transição ${ }^{4} \mathrm{~F}_{3 / 2} \rightarrow{ }^{4} \mathrm{I}_{15 / 2}$ não foi detectada e teve sua energia atribuída de acordo com os espectros de absorção.

\begin{tabular}{|c|c|c|c|c|c|c|c|}
\hline & $\begin{array}{l}\Omega_{2}\left(\mathbf{p m}^{2}\right) \\
3,3 \pm 0,3\end{array}$ & & $\begin{array}{l}\Omega_{4}(\mathbf{p} \\
4,4 \pm\end{array}$ & & $\begin{array}{l}\Omega_{\mathbf{6}}(\mathrm{I} \\
2,0 \pm\end{array}$ & $\begin{array}{l}\left.\mathbf{m}^{\mathbf{2}}\right) \\
0,1\end{array}$ & \\
\hline & A & $-1)$ & & & $\beta$ & & \\
\hline${ }^{4} \mathbf{I}_{9 / 2}$ & ${ }^{4} \mathbf{I}_{11 / 2}$ & ${ }^{4} \mathbf{I}_{13 / 2}$ & ${ }^{4} \mathbf{I}_{15 / 2}$ & ${ }^{4} \mathbf{I}_{9 / 2}$ & ${ }^{4} \mathbf{I}_{\mathbf{1 1 / 2}}$ & ${ }^{4} \mathbf{I}_{13 / 2}$ & ${ }^{4} \mathbf{I}_{15 / 2}$ \\
\hline 1014 & 1746 & 303 & 12 & 0,35 & 0,55 & 0,09 & 0,006 \\
\hline
\end{tabular}

Reisfeld e Jorgensen ${ }^{17}$ consideram que $\Omega_{2}$ indica a covalência da ligação entre o íon TR e a rede, quanto maior o valor de $\Omega_{2}$ maior será a covalência da ligação e menor a simetria ao redor do íon . Tanabe et al., relacionaram altos valores de $\Omega_{2}$ com um aumento da assimetria ao redor do íon $\mathrm{TR}^{18}$. No entanto Uhlmann, et al. ${ }^{16}$, afirmam que nem sempre para vidros aluminatos estas regras são observadas, sem que para isso atribuíssem uma causa.

Outros parâmetros espectroscópicos como probabilidade de emissão espontânea $(A)$; tempo de vida radiativo $\left(\tau_{0}\right)$; eficiência quântica $(\eta)$; razão de ramificação $(\beta)$ e seção eficaz de emissão $(\sigma)$, podem ser obtidos a partir dos valores de $\Omega_{2}, \Omega_{4}$ e $\Omega_{6}$.

A probabilidade de emissão espontânea $(A)$ é dada pela expressão:

$A=\frac{64 \pi^{4} E^{3}}{3(2 J+1) h} \frac{n\left(n^{2}+2\right)^{2}}{9} e^{2} \sum_{\lambda=2,4,6} \Omega_{\lambda}\left|\left\langle a J\left\|U^{\lambda}\right\| b J^{\prime}\right\rangle\right|^{2}$

sendo, $E$ a energia da transição $\left(\mathrm{em}^{-1}\right)$ e $J$ o número quântico de momento angular total do nível emissor.

Se a transição radiativa será ou não observada num espectro dependerá dos diferentes caminhos de desativação do nível em questão. Esta pode apresentar uma alta probabilidade radiativa, mas também pode ocorrer uma desativação via fônons ou transferência de energia, dependendo da composição da matriz. Os valores calculados para a probabilidade de emissão espontânea para as transições ${ }^{4} \mathrm{~F}_{3 / 2} \rightarrow{ }^{4} \mathrm{I}_{9 / 2},{ }^{4} \mathrm{~F}_{3 / 2} \rightarrow{ }^{4} \mathrm{I}_{11 / 2},{ }^{4} \mathrm{~F}_{3 / 2} \rightarrow{ }^{4} \mathrm{I}_{13 / 2}$ e ${ }^{4} \mathrm{~F}_{3 / 2} \rightarrow{ }^{4} \mathrm{I}_{15 / 2}$ do íon $\mathrm{Nd}^{3+}$ no vidro CASM estão na Tabela 1 . As probabilidades de emissão espontânea calculadas não variam com a concentração de neodímio.

A partir das probabilidades de emissão espontânea de um certo nível, pode-se calcular o tempo de vida radiativo para este através da expressão:

$\tau_{0}=\frac{1}{\sum_{S, L, J} A\left(a J ; b J^{\prime}\right)}$

sendo, o termo no denominador a soma das probabilidades de emissão espontânea para todos os níveis abaixo do nível emissor (no caso o ${ }^{4} \mathrm{~F}_{3 / 2}$ ). Na maioria dos casos, $\tau_{0}$ é maior ou igual ao tempo de vida experimental $(\tau)$, pois ele corresponde à um sistema onde não ocorre transferência de energia e decaimento multifônons. Para os vidros CASM, os valores dos tempos de vida experimentais do nível ${ }^{4} \mathrm{~F}_{3 / 2}$, a 300 e $77 \mathrm{~K}$, apresentam uma diminuição com o aumento da concentração de neodímio (Figura 3). Na mesma figura são mostrados os valores do tempo de vida calculados pelo modelo de Judd-Ofelt $\left(\tau_{0}\right)$. A diferença entre os valores dos tempos de vida a 300 e $77 \mathrm{~K}$, assim como a diminuição destes valores com o aumento da concentração de $\mathrm{Nd}^{3+}$, são atribuídas a processos de desativação não-radiativos (via fônons, transferência de energia, etc.).

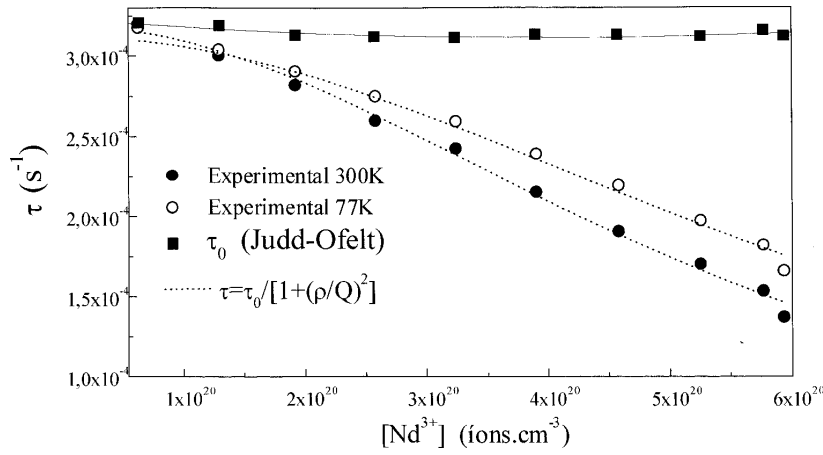

Figura 3. Tempo de vida calculado pelo modelo de Judd-Ofelt e variação dos tempos de vida experimentais da transição ${ }^{4} F_{3 / 2} \rightarrow{ }_{11 / 2}^{4}(1076 \mathrm{~nm})$ em função da temperatura e da concentração de $\mathrm{Nd}^{+3}$. Laser de bombeio $514 n m$.

Stokowski, et al $^{19}$, estudaram os processos de transferência de energia entre íons $\mathrm{Nd}^{3+}$ e estabeleceram uma relação empírica para o tempo de vida:

$$
\tau=\frac{\tau_{0}}{1+\left(\frac{\rho}{Q}\right)^{n}}
$$

sendo $\tau$ o tempo de vida esperado para uma determinada concentração de TR $(\rho)$; $\tau_{0}$ é o tempo de vida para um sistema diluído onde não há transferência de energia; $Q$ a concentração de supressão (para a qual o tempo de vida experimental foi reduzido à metade de $\tau_{0}$ - no caso $4 \%$ de $\mathrm{Nd}_{2} \mathrm{O}_{3}$ ou $5,25.10^{20}$ íons.cm ${ }^{-3}$ ); e $n$ é um parâmetro que depende do íon TR $\left(n=2 \text { para o } \mathrm{Nd}^{3+}\right)^{10}$.

A equação (6) foi usada para o ajuste das curvas dos tempos de vida experimentais da Figura 3, e estão representadas pelas linhas pontilhadas.

Um parâmetro que pode ser obtido a partir dos tempos de vida experimental e calculado é a eficiência quântica de uma dada transição. Ela depende da relação entre $\tau$, onde são considerados processos radiativos e não radiativos, e $\tau_{0}$.

$$
\eta_{\text {calc. }}=\frac{\tau}{\tau_{0}}
$$

Os resultados de $\eta_{\text {calc. }}$ são apresentados na Figura 4.

Baesso, et $\mathrm{al}^{20}$, através da técnica de lente térmica mediram a eficiência quântica das mesmas amostras de vidro CASM aqui estudadas, e os resultados obtidos estão em boa concordância com os calculados pelo modelo de Judd-Ofelt apresentados neste trabalho.

Outro parâmetro importante é a razão de ramificação. Este parâmetro mede a relação entre as intensidades das transições eletrônicas que ocorrem a partir de um mesmo nível. Neste sistema o nível de interesse é o ${ }^{4} \mathrm{~F}_{3 / 2}$, nível de partida para as 


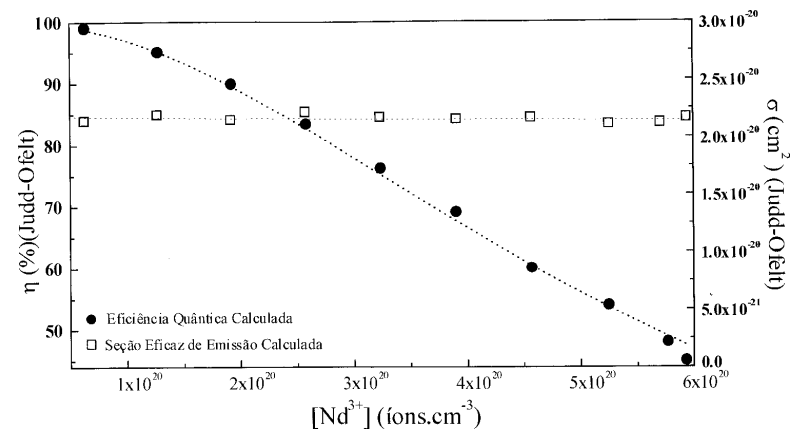

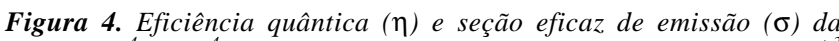
transição ${ }^{4} F_{3 / 2} \rightarrow{ }^{4} I_{11 / 2}(1076 \mathrm{~nm})$, em função da concentração de $\mathrm{Nd}^{+3}$ na matriz CASM. Os valores foram obtidos das equações 7 e 9.

transições ${ }^{4} \mathrm{~F}_{3 / 2} \rightarrow{ }^{4} \mathrm{I}_{9 / 2}(900 \mathrm{~nm}),{ }^{4} \mathrm{~F}_{3 / 2} \rightarrow{ }^{4} \mathrm{I}_{11 / 2}(1076 \mathrm{~nm}),{ }^{4} \mathrm{~F}_{3 / 2} \rightarrow{ }^{4} \mathrm{I}_{13 / 2}$ (1300nm) e ${ }^{4} \mathrm{~F}_{3 / 2} \rightarrow{ }^{4} \mathrm{I}_{15 / 2}(1800 \mathrm{~nm})$. A razão de ramificação depende das probabilidades de emissão espontânea, as quais dependem do índice de refração e dos valores dos parâmetros de intensidade de Judd-Ofelt, e é dada pela expressão:

$$
\beta\left(b J^{\prime} ; \bar{a} \bar{J}\right)=\frac{A\left(b J^{\prime} ; \bar{a} \bar{J}\right)}{\sum_{J} A\left(b J^{\prime} ; a J\right)}
$$

sendo, $A\left(b J^{\prime} ; \overline{a J}\right)$ a probabilidade de emissão espontânea do nível $b J^{\prime} \rightarrow a J^{\prime} e \sum A\left(b J^{\prime} ; a J\right)$ e a probabilidade de emissão espontânea total.

$\mathrm{Na}$ referência 21 pode-se encontrar a figura que mostra a variação de $\beta$ em função da razão $\Omega_{4} / \Omega_{6}$ em escalas logarítmicas, para diferentes sistemas dopados com $\mathrm{Nd}^{3+21}$. Em nosso sistema a relação $\log \left(\Omega_{4} / \Omega_{6}\right) \cong 0,4$, indicando que a transição ${ }^{4} \mathrm{~F}_{3 / 2} \rightarrow{ }^{4} \mathrm{I}_{11 / 2}(1076 \mathrm{~nm})$ deve ser a mais intensa. Estas indicações estão de acordo com as observações experimentais.

A seção eficaz de emissão é proporcional ao comprimento de onda e a intensidade da banda de fluorescência e, inversamente proporcional à largura de linha efetiva:
$\sigma\left(J ; J^{\prime}\right)=\frac{\lambda_{p}^{4}}{8 \pi c n^{2} \Delta \lambda_{\text {efe }}} A\left[\left({ }^{4} F_{3 / 2},{ }^{4} I_{j}\right)\right]$

sendo, $\lambda_{p}$ o comprimento de onda do pico de emissão e $\Delta \lambda_{e f e}$. a razão entre a área da banda e sua intensidade (largura de linha efetiva). Os valores obtidos para os vidros CASM são apresentados na Figura 4.

A Tabela 2 mostra o comprimento de onda referente a transição ${ }^{4} \mathrm{~F}_{3 / 2} \rightarrow{ }^{4} \mathrm{I}_{11 / 2}$ e em diversas matrizes vítreas. A dependência da posição do pico de emissão com a composição da matriz é devida ao efeito nefelauxético. Um aumento na covalência da ligação, faz com que a interação entre os elétrons seja reduzida, pois estes se "espalham" mais pelos orbitais ligantes. Consequentemente, as transições eletrônicas entre estados nos quais a diferença de energia é determinada pela interação eletrônica, terão suas emissões deslocadas para comprimentos de onda maiores (energias menores). Na Tabela 2 vemos que o vidro CASM, dentre os vidros óxidos ali comparados, apresenta a maior covalência da ligação Nd-O.

A largura de linha efetiva depende dessa variação na covalência da ligação $\mathrm{Nd}-\mathrm{O}$, pois essa variação é um indicador da força do campo ao redor do íon TR. Um estreitamento da largura de linha é observado quando íons que geram um campo fraco estão presentes na matriz. Por outro lado, íons que geram um campo forte provocam um alargamento da linha, indicando uma assimetria na esfera de coordenação. Portanto, uma linha larga indica a presença de um campo local forte, ou uma variação deste campo de sítio para sítio do TR (grande desordem). Dentro de sistemas formadores de vidros, a largura efetiva de linha aumenta com o aumento da carga e com a diminuição do tamanho do cátion modificador.

Para situar as qualidades espectroscópicas da matriz do vidro CASM dentre outras, a Tabela 3 a compara com matrizes semelhantes e com $\mathrm{YAG}^{22}$ e ED-2 ${ }^{23}$, que são meios ativos comerciais para laseres. Outra comparação pode ser realizada com os vidros estudados na referência 24 , que também apresenta os resultados dos parâmetros de Judd-Ofelt para uma matriz de composição semelhante à do vidro CASM.

Tabela 2. Posição da linha ${ }^{4} \mathrm{~F}_{3 / 2} \rightarrow{ }^{4} \mathrm{I}_{11 / 2}$ do $\mathrm{Nd}^{3+}$ em diferentes matrizes vítreas ${ }^{15}$.

\begin{tabular}{|c|c|c|c|c|c|}
\hline Matriz (Vidros) & Fosfato & Borato & Germanato & Aluminato & CASM \\
\hline $\begin{array}{c}\lambda(\mathrm{nm}) \\
\left({ }^{4} \mathrm{~F}_{3 / 2} \rightarrow{ }^{4} \mathrm{I}_{11 / 2}\right)\end{array}$ & $1052-1057$ & $1054-1062$ & $1060-1063$ & 1069 & 1076 \\
\hline
\end{tabular}

Tabela 3. Parâmetros de intensidade de Judd-Ofelt para diferentes matrizes: CANB (vidro óxido, composto por $\mathrm{Ca}^{2+}, \mathrm{Al}^{3+}, \mathrm{Na}^{+} \mathrm{e}^{\mathrm{a}}$ $\left.\mathrm{Ba}^{2+}\right)^{15}$; CANS (vidro composto por $\mathrm{Ca}^{2+}, \mathrm{Al}^{3+}, \mathrm{Na}^{+}$e $\mathrm{Sr}^{2+}$ ) ${ }^{15}$, CASM (o vidro em estudo neste trabalho); cristal $\mathrm{CaZn}_{2} \mathrm{Y}_{2} \mathrm{Ge}_{3} \mathrm{O}_{12}{ }^{22}$; YAG (ytrium aluminium garnet) ${ }^{23}$; ED-2 é um vidro laser comercial à base de silicato ${ }^{15}$.

\begin{tabular}{|c|c|c|c|c|c|c|c|}
\hline Matriz & $\begin{array}{c}\Omega_{2} \\
\left(\mathrm{pm}^{2}\right)\end{array}$ & $\begin{array}{c}\Omega_{4} \\
\left(\mathrm{pm}^{2}\right)\end{array}$ & $\begin{array}{c}\Omega_{6} \\
\left(\mathrm{pm}^{2}\right)\end{array}$ & $\begin{array}{c}\lambda(\mathrm{nm}) \\
{ }^{4} \mathrm{~F}_{3 / 2} \rightarrow{ }^{4} \mathrm{I}_{11 / 2}\end{array}$ & $\begin{array}{l}\tau_{\exp } \\
(\mu s)\end{array}$ & $\begin{array}{l}\sigma\left(\mathbf{c m}^{2}\right) \\
\left(x 10^{-20}\right)\end{array}$ & $\eta_{\text {calc }}$ \\
\hline $\begin{array}{l}\text { CANB } \\
\left(0,5 \% \mathrm{Nd}_{2} \mathrm{O}_{3}-\mathrm{mol}\right)\end{array}$ & $\begin{array}{c}4,4 \\
\pm 0,4\end{array}$ & $\begin{array}{c}5,2 \\
\pm 0,5\end{array}$ & $\begin{array}{c}2,7 \\
\pm 0,2\end{array}$ & 1068 & 277 & $1,3 \pm 0,2$ & 0,80 \\
\hline $\begin{array}{l}\text { CANS } \\
\left(0,5 \% \mathrm{Nd}_{2} \mathrm{O}_{3}-\mathrm{mol}\right)\end{array}$ & $\begin{array}{c}3,7 \\
\pm 0,3\end{array}$ & $\begin{array}{c}5,0 \\
\pm 0,5\end{array}$ & $\begin{array}{c}2,9 \\
\pm 0,2\end{array}$ & 1075 & 260 & $1,3 \pm 0,2$ & 0,75 \\
\hline $\begin{array}{l}\text { CASM } \\
\left(2,5 \% \mathrm{Nd}_{2} \mathrm{O}_{3}-\text { massa }\right)\end{array}$ & $\begin{array}{c}3,3 \\
\pm 0,3\end{array}$ & $\begin{array}{c}4,4 \\
\pm 0,3\end{array}$ & $\begin{array}{c}2,0 \\
\pm 0,1\end{array}$ & 1076 & $\begin{array}{l}242 \\
\pm 10\end{array}$ & $2,2 \pm 0,1$ & $\begin{array}{c}\mathbf{0 , 7 8} \\
\pm \mathbf{0 , 0 5}\end{array}$ \\
\hline $\begin{array}{l}\text { Cristal } \mathrm{CaZn}_{2} \mathrm{Y}_{2} \mathrm{Ge}_{3} \mathrm{O}_{12} \\
\left(1 \% \mathrm{Nd}^{+3} \mathrm{~mol}\right)\end{array}$ & 0,98 & 3,20 & 3,63 & 1058 & $\begin{array}{l}285 \\
\pm 10\end{array}$ & - & - \\
\hline $\begin{array}{l}\text { YAG: } \mathrm{Nd}^{+3} \\
(0,8 \% \mathrm{~mol})\end{array}$ & 0,20 & 2,70 & 5,00 & 1064 & $\begin{array}{l}240 \\
\pm 10\end{array}$ & 28,0 & $0,91-0,99$ \\
\hline ED-2 & 3,30 & 4,68 & 5,18 & 1064 & 310 & 2,71 & 0,83 \\
\hline
\end{tabular}




\section{Luminescência no Infravermelho}

Os espectros de luminescência na região do IV, a 300 e $77 \mathrm{~K}$, para uma amostra com $1 \%$ de $\mathrm{Nd}_{2} \mathrm{O}_{3}$, são apresentados na Figura 5. As transições observadas são aquelas do nível emissor ${ }^{4} \mathrm{~F}_{3 / 2}$ para: ${ }^{4} \mathrm{I}_{9 / 2}(900$ e $920 \mathrm{~nm}) ;{ }^{4} \mathrm{I}_{11 / 2}(1076$ e $1120 \mathrm{~nm})$; ${ }^{4} \mathrm{I}_{13 / 2}(1350$ e $1420 \mathrm{~nm})$, e estão esquematizadas na Figura $6 \underline{\mathrm{A}}$. A transição ${ }^{4} \mathrm{~F}_{3 / 2} \rightarrow{ }^{4} \mathrm{I}_{15 / 2}$, esperada em $\cong 1800 \mathrm{~nm}$, não foi observada devido a sua probabilidade de transição ser muito pequena (ver Tabela 1) e também pela possibilidade de sua energia ser reabsorvida pela transição ${ }^{4} \mathrm{I}_{9 / 2} \rightarrow{ }^{4} \mathrm{I}_{15 / 2}$ (Figura $\left.6 \underline{\mathrm{B}}\right)^{25}$. Além disso, o sistema de detecção empregado apresenta baixa sensibilidade na região de $1800 \mathrm{~nm}$.

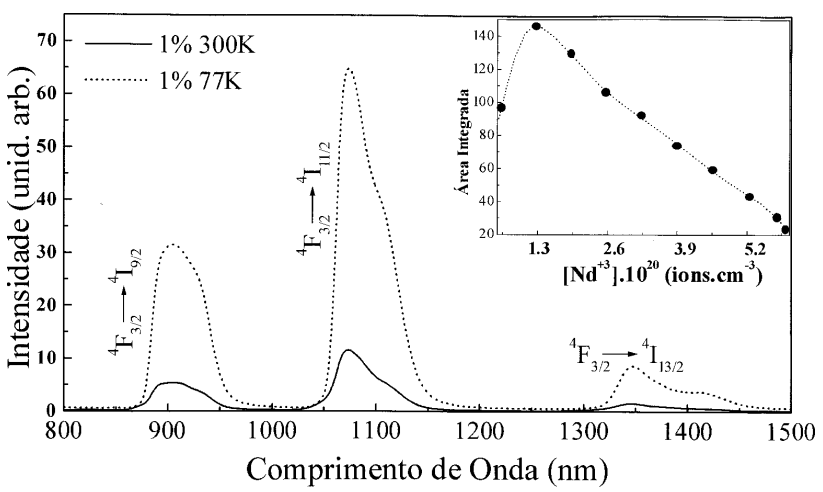

Figura 5. Espectros de luminescência na região do IV a 300 e $77 \mathrm{~K}$ para a amostra com $1 \%$ de $\mathrm{Nd}_{2} \mathrm{O}_{3}$. No destaque, a variação da área integrada sob a banda da transição em 1076nm em função da concentração de $\mathrm{Nd}^{3+}$. Laser de bombeio - 514nm.

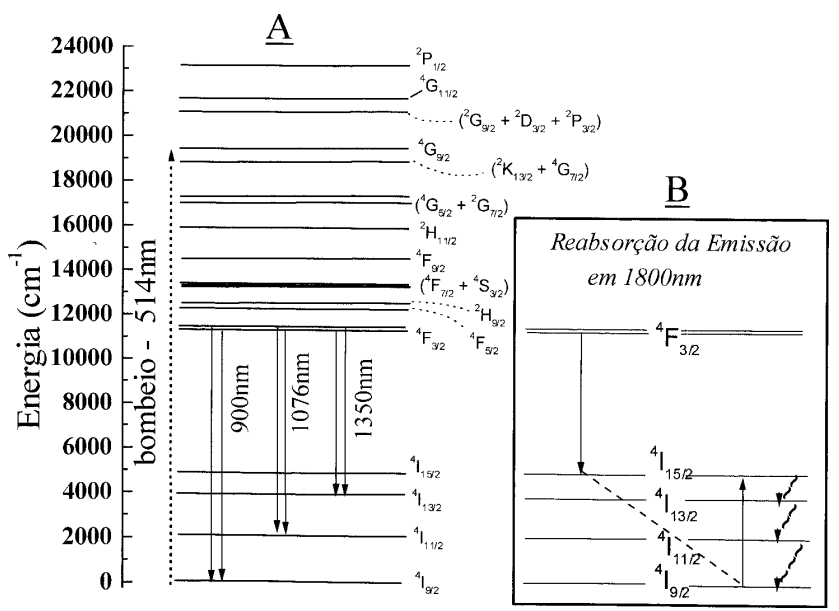

Figura 6. Diagrama de níveis de energia do $\mathrm{Nd}^{+3}$ no vidro CASM, construído a partir dos espectros de absorção, mostrando as transições no $I V(\underline{A})$ e o processo de reabsorção da emissão em $\cong 1800 \mathrm{~nm}$ no $N d^{+3}(\underline{B})$.

A intensidade da luminescência do íon TR no IV depende da concentração e da composição da matriz. No primeiro caso, processos de relaxação-cruzada diminuem a população do nível ${ }^{4} \mathrm{~F}_{3 / 2}$ e consequentemente as intensidades das emissões em 900,1076 e $1350 \mathrm{~nm}$ e o tempo de vida deste nível. A eficiência desses processos de transferência de energia (TE) varia com o inverso da potência da distância $\left(1 / \mathrm{R}^{\mathrm{n}}\right)^{26}$, onde o valor de $n$ pode ser 6,8 ou 10 , dependendo da natureza do mecanismo da TE. No destaque da Figura 5 vemos a variação da área integrada sob a banda de emissão em $1076 \mathrm{~nm}\left({ }^{4} \mathrm{~F}_{3 / 2} \rightarrow{ }^{4} \mathrm{I}_{11 / 2}\right)$, que diminui a partir de $1 \%$ de $\mathrm{Nd}_{2} \mathrm{O}_{3}$. Isso é atribuído aos processos de relaxação cruzada. A ocorrência de relaxação cruzada também depende dos níveis envolvidos na excitação da amostra. Quando o bombeio é realizado em $800 \mathrm{~nm}$, a diminuição na área integrada da banda em $1076 \mathrm{~nm}$, ou seja, a supressão da luminescência, passa a ocorrer a partir de $2,5 \%$ de $\mathrm{Nd}_{2} \mathrm{O}_{3}$.

A dependência com a matriz será de acordo com a energia de fônon máxima que esta apresentar. No caso dos vidros CASM esta energia é de $\cong 800 \mathrm{~cm}^{-1}$ e sua influência é observada nas linhas de emissão na região do visível. Os espectros da região entre 520 e $760 \mathrm{~nm}$ foram obtidos pela excitação do nível ${ }^{4} \mathrm{G}_{9 / 2}(514 \mathrm{~nm})$, e não foram observadas emissões nessa região. A população deste nível decai rapidamente via fônons para os níveis abaixo dele. Segundo Buisson et al. ${ }^{27}$, o tempo de vida para o ${ }^{4} \mathrm{G}_{5 / 2}$ em $\mathrm{LaF}_{3}$ é de $\cong 100$ ns e os íons excitados levam menos que $1 \mu$ s para atingir o nível metaestável ${ }^{4} \mathrm{~F}_{3 / 2}$. No caso do vidro CASM isso fica evidente se observarmos na Figura $6 \underline{\mathrm{A}}$ que, a diferença de energia entre a maioria dos níveis abaixo do ${ }^{4} \mathrm{G}_{9 / 2}$ é de $\cong 1000 \mathrm{~cm}^{-1}$.

\section{CONCLUSÕES}

O vidro CASM com baixa quantidade de sílica e com até $5 \%$ de $\mathrm{Nd}_{2} \mathrm{O}_{3}$, apresentou transmissão na região do IV até $4,5 \mu \mathrm{m}$. O processo de relaxação-cruzada do íon $\mathrm{Nd}^{3+}$ diminuiu a eficiência da emissão em $1076 \mathrm{~nm}$ quando do aumento da concentração deste íon. Dentre as amostras analisadas, aquelas com concentração de $\mathrm{Nd}_{2} \mathrm{O}_{3}$ entre 0,5 e 1,5(\% massa) são as que apresentam melhores valores de tempo de vida, luminescência e eficiência quântica, a partir da excitação em $514 \mathrm{~nm}$. Quando comparado diretamente com o vidro ED-2, que é um meio ativo comercial para laser de alta potência, observamos que as características espectroscópicas são semelhantes.

Os vidros CASM são promissores para serem utilizados quer como meio ativo para laseres na região do infravermelho.

\section{AGRADECIMENTOS}

À FAPESP pelo apoio financeiro e pela bolsa de pós-graduação concedida a Édison Pecoraro.

\section{REFERÊNCIAS}

1. Hafner, H. C.; Kreidl, N. J. and Weidel, R. A.; J. Am. Ceram. Soc. 1958, 41, 315.

2. Davy, J. R.; Glass Technol. 1978 April, 19(2).

3. Bartholomew, R. F.; Aitken, B. G. and Newhouse, M. A.; J. Non-Cryst. Sol. 1995, 184, 229.

4. Uhlmann, E. V.; Weinberg, M. C.; Kreidl, N. J and Goktas, A. A.; J. Am. Ceram. Soc. 1993, 76, 449.

5. Baesso, M. L.; Bento, A. C.; Duarte, A. R.; Neto, A. M.; Miranda, L. C. M.; Sampaio, J. A.; Catunda, T.; Gama, S.; Gandra, F. C. G.; J. Appl. Phys. 1999, 86, 3144

6. Hecht, J.; Entendendo Fibras Óticas, Ed. Berkeley (RJ), traduzido por Roberto R. Tavares, 1993, p. 201.

7. King, W. A. and Shelby, J. E.; Phys. Chem. Glasses 1996, 37, 1.

8. Carnall, W. T.; Fields, P. R. and Rajnak, K.; J. Chem. Phys. 1968, 49, 4424.

9. Judd, B. R.; Phys. Rev. 1962, 128, 750.

10. Ofelt, G. S.; J. Chem. Phys. 1962, 37, 511.

11. Saisudha, M. B. and Ramakrishna, J.; Phys. Rev. B 1996, 53, 6186

12. Payne, S. A.; Marshall, C. D.; Bayramian, A.; Wilke, G. D. and Hayden, J. S.; Appl. Phys. B 1995, 61, 257.

13. Kaminskii, A. A.; Boulon, G.; Buoncristisni, M.; Di Bartolo, B.; Kornienko, A. and Mironov, V.; Phys. Stat. Sol. (A) 1994, 141, 471

14. Guyot, Y.; Garapon, C. and Moncrogé, R.; Optical Mat. 1994, 3, 275.

15. Carnall, W. T.; Fields, P. R. and Rajnak, K.; J. Chem. Phys. 1968, 49, 4412. 
16. Uhlmann, E. V.; Weinberg, M. C.; Kreidl, N. J; Burgner, L. L.; Zanoni, R. and Church, K. H.; J. Non-Cryst. Sol. 1994, 178, 15.

17. Reisfeld, R. and Jørgensen, C. K., in: Handbook on the Physics and Chemistry of Rare Earth, ed K. A. Gschneidner and L. Eyring, North Holland, Amsterdam, 1984.

18. Tanabe, S.; Ohyagi, T.; Soga, N.; Hanada, T.; Phys. Rev. $B$ 1992, 46, 3305 .

19. Stokowski, S. E.; Saroyan, R. A. and Weber, M. J. - Nddoped laser glass spectroscopic and physical properties, Lawrence Livermore National Laboratory, M-095, Rev.2, November, 1981, in:Rare earth doped fiber lasers and amplifiers, Edited by Michel J. F. Digonnet, Ed. Marcel Dekker, Inc., 1993.

20. Baesso, M. L.; Bento, A. C.; Andrade, A. A.; Catunda, T.; Sampaio, J. A. and Gama, S.; J. Non Cryst. Sol. 1997, 219, 165.
21. Brown, D. C.; "High-Peak-Power Nd:Glass Laser Systems" - Springer Series in Optical Sciences; Ed. Springer -Verlag, 1981, 25, p. 23.

22. Sardar, D. H.; Vizcarra, S.; Islam, M. A.; Allik, H. T.; Sharp, E. J. and Pinto, A. A.; Optical Mat. 1994, 3, 257.

23. Krupke, W. F.; IEEE J. Quantum Eletron. 1971, QE7, 153.

24. Jacobs, R. R.; Weber, M. J.; IEEE J. Quantum Electron. 1976, $Q E-12,102$.

25. Miniscalco, W. J.; Optical and Electronic Properties of Rare Earth Ions in Glasses - in: Rare Earth Doped Fiber Lasers and Amplifiers, Digonet, M. J. F., Ed. Marcel Dekker, 1993, p.44.

26. Dexter, D. L. and Schulman, J. H.; J. Chem. Phys. 1954, 22, 1063 .

27. Buisson, R. and Liu, J. Q.; J. Physique 1984, 45, 1523. 